\title{
Hopping and running on two legs or four
}

from R. McNeill Alexander

Most mammals run on all fours but some run on just two legs (like men) or hop (like kangaroos). Some birds run, some hop and some do both. What are the relative merits of the different gaits? Do they differ in energy cost?

Energy costs of running and hopping can be inferred from measurements of oxygen consumption. Some of the difficulties of physiological experimentation on moving animals can be avoided by training the animal to run on a moving belt as shown. Data has been collected for mammals ranging from mice to horses and lions, and for many birds (summarized by Fedak \& Seeherman Nature 282, 713; 1979). Nearly all of it conforms to a simple rule: the power $P$ required for level running at speed $u$ is given by

$$
P(u)=P(0)+k u
$$

where $k$ is a constant for the particular animal. $P(0)$ is a little more than the power consumption at rest because it includes a component for maintenance of posture.

Equation (1) implies that the energy required to travel a given distance (in excess of the requirement for standing still) is more or less independent of speed.

This may seem remarkable, but experiments with red kangaroos gave an even more remarkable result (Dawson \& Taylor Nature 246, 313; 1973). The power consumption of a hopping kangaroo is independent of speed, or may even fall slightly as speed increases. The energy used by a kangaroo travelling a given distance therefore falls steeply as speed increases. At low speeds a kangaroo uses far more power than (for instance) a gazelle of the same mass, but at $5 \mathrm{~m} \mathrm{~s}^{-1}$ the two animals use about equal powers. It has been suggested that at higher speeds, a kangaroo might travel more economically than a gazelle, but it is difficult to measure power consumption at high speeds because the animals build up oxygen debts.

Various rodents hop like small kangaroos. There are kangaroo rats (Dipodomys) in N. America, springhares (Pedetes) in Africa and hopping mice (Notomys) in Australia. Is power independent of speed for them all? Thompson, McMillen, Burke \& Taylor (this issue of Nature, p223) find that it is not. Equation (1) holds for many and probably all hopping rodents, with values of $k$ about the same as for quadrupeds of similar mass. The same is true for the rat kangaroo (Bettongia, a marsupial). The large kangaroos seem to stand alone in their peculiar energetics.

Though equation ( 1 ) holds for a wide variety of animals, the constant $k$ is

R. McNeill Alexander is Professor of Zoologyat the University of Leeds.

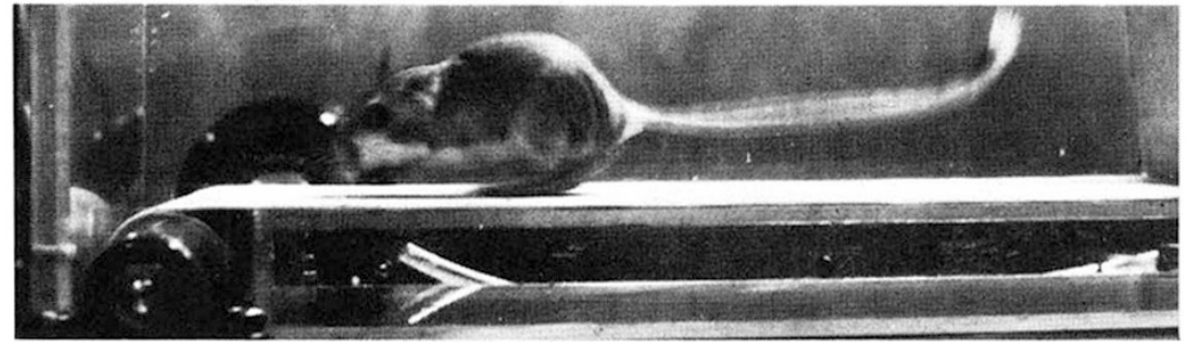

Dipodomys deserti hopping on a moving belt to assess energy consumption. Experimental details are given by Thompson et al. on p.223 of this issue of Nature. different for animals of different sizes. Until recently the data seemed to indicate that $k$ was proportional to (body mass) (b. $^{0.6}$ for quadrupeds but to (body mass) ) $^{0.8}$ for bipeds. Consequently bipedalism was more economical than quadrupedalism for small animals, but less economical for large ones. Why, then, are there small quadrupeds (such as mice) and large bipeds (such as ostriches and, formerly, tyrannosaurs)?

This apparent paradox seems to have been an artefact of the sample of species originally selected for study. Fedak \& Seeherman (Nature 282, 713; 1979) showed that though penguins and geese need twice as much power for running as dogs of the same mass, ostriches are as economical as ponies. For any body mass, a wide range of values of $k$ is possible, but the general trend both for quadrupeds and for bipeds is for $k$ to be proportional to (body mass) ${ }^{0.7}$.

It would be satisfying to be able to explain why kangaroos and smaller hoppers have different relationships between power and speed, and why $k$ tends to be proportional to (body mass) ${ }^{0.7}$. This has not yet been achieved. Calculations based on the mechanical work required of the leg muscles provide satisfactory explanations of the power requirements of kangaroos and also of quadrupedal mammals of similar size, and show that slow hopping is more expensive than running because it involves larger fluctuations of potential energy (Alexander \& Goldspink Mechanics and energetics of animal locomotion, 1977).

Similar calculations grossly underestimate the power requirements of small runners and hoppers, such as mice and quail: indeed they predict that $k$ should be proportional to (body mass) $)^{1.0}$. Taylor, Heglund, McMahon \& Looney (J. exp. Biol. 86, 9; 1980) have recently tried to resolve this discrepancy, by suggesting that the main metabolic energy cost of running is associated with the generation of muscular force, rather than the performance of work. The forces required are proportional to body mass but smaller mammals need faster muscles, with shorter cross-bridge cycling times, so exertion of force may be more expensive for them. Taylor and his colleagues show for rats, dogs, men and horses, that carrying a load increases $k$ in the same proportion as it increases the forces on the feet. This is consistent with their hypothesis but is not clear evidence for it (as they themselves admit), because the experiment increases forces and work in the same proportion. The hypothesis is unlikely to win general acceptance unless it can be supported by less ambiguous evidence and shown to be quantitatively consistent with our knowledge of muscle physiology.
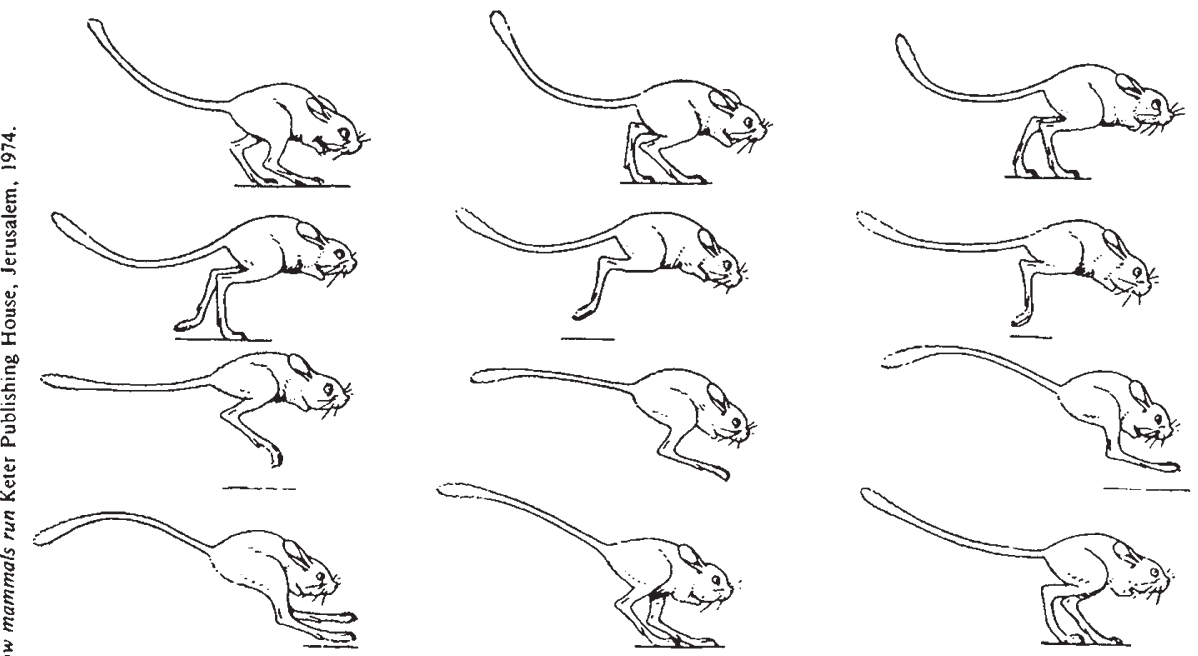

One of the hopping rodents (a jerboa, Allactaga severtzovi). The action is like a kangaroo but the energetics may be quite different. 